\section{Calidad de las investigaciones basadas en encuestas: directrices para buenos informes}

\section{Survey-based investigations quality: guidelines for good reports}

\section{Resumen}

La evaluación de la calidad de los estudios es muy importante en las diferentes disciplinas científicas. Los estudios basados en encuestas al igual que otros tipos de estudio requieren altos estándares de calidad tanto en su ejecución como en su informe, sin embargo, existe evidencia que indica que no es así. Se han propuesto muchas herramientas que ayudan a valorar la calidad del informe de los estudios que utilizan encuestas, la gran mayoría no están validadas ni cuentan con un consenso general para su uso; por lo cual, el objetivo de esta nota científica es presentar las directrices más aceptadas (SURGE y CHERRIES) que ayuden a informar este tipo de estudios de manera eficaz y transparente.

Palabras clave: Encuestas y cuestionarios; Calidad metodológica; Encuestas de salud bucal; Estudios epidemiológicos; Encuestas de salud (fuente: DeCS BIREME).

\begin{abstract}
Studies quality evaluation is very important in the different scientific disciplines. Survey-based studies as well as other study types require high quality standards both in their execution and in their report, however, there is evidence indicating that it is not so. Many tools have been proposed to help assessing the survey-based reports quality, the vast majority neither are validated nor have a general consensus for their use; Therefore, the aim of this scientific note is to present the most accepted guidelines (SURGE and CHERRIES) that help inform this type of studies in an effective and transparent way.
\end{abstract}

Keywords: Surveys and questionnaires; Methodological quality; Dental health surveys; Epidemiologic studies; Health surveys (source: MeSH NLM).

\section{Nota Científica}

Manuel Antonio Mattos-Vela ${ }^{1, a,}$ Dahiana Alfaro Carballido ${ }^{2, b}$

${ }^{1}$ Universidad Nacional Mayor de San Marcos, Facultad de Odontología. Lima, Perú.

2 Universidad de San Martín de Porres, Facultad de

Odontología, Lima, Perú.

a Doctor en Estomatología.

${ }^{\mathrm{b}}$ Magíster en Estomatología.

\section{Correspondencia:}

Manuel Antonio Mattos-Vela: mmattosv@unmsm.edu.pe Facultad de Odontología, UNMSM. Calle Germán Amézaga 375. Lima 1, Perú.

ORCID: 0000-0002-5701-1961

\section{Coautora:}

Dahiana Alfaro Carballido: alfaro.dahiana@gmail.com ORCID: 0000-0001-6310-8316

\section{Editor:}

Juan Carlos Cuevas-González

Universidad Autónoma de Ciudad Juárez, México.

Conflicto de intereses: los autores declaran ser miembros del Comité Editor de la revista Odontología Sanmarquina.

Fuente de financiamiento: autofinanciado.

Recibido: 24/09/19

Aceptado: 18/12/19

Publicado: 24/02/20 


\section{Introducción}

Las encuestas son un método de investigación bastante utilizado en Odontología en sus diferentes especialidades, pero en mayor proporción en el área de salud pública, ética y educación; aunque las demás especialidades no se ven excluidas, puesto que es bastante común su uso en la etapa inicial de ensayos clínicos en el reclutamiento de pacientes ${ }^{1}$. Este método puede aplicarse tanto en investigación cuantitativa, cualitativa o mixta; recolecta la información a través de cuestionarios, sobre un tema específico, pudiendo generalizarse sus resultados a poblaciones mayores; además, son de fácil aplicación y bajo coste ${ }^{2}$.

Las investigaciones que utilizan encuestas son igual de importantes que cualquier otro tipo de investigaciones, son el inicio en estudios exploratorios, así como ejes transversales en investigación cuantitativa. Son la base para poder pasar a los siguientes niveles de evidencia, permitiendo así, dar un enfoque integral a la investigación en salud donde no solo se valora la parte cuantitativa del proceso salud-enfermedad, sino que nos permite darle un enfoque más holístico integrando el aspecto biopsicosocial del ser humano. Además, las encuestas son útiles para abordar temas que son difíciles de evaluar; permitiendo construir constructos específicos al tema que se desea investigar ${ }^{3,4}$. Las principales limitaciones de este método son los sesgos de información y de medición que tienen que ver directamente con la elección y elaboración del instrumento, siendo el punto crítico la validez y confiabilidad, las que afectan directamente la generalización de los resultados de este tipo de estudios ${ }^{5,6}$.

En una investigación realizada por Bennett $e t$ al. ${ }^{3}$ sobre la evaluación de la calidad de informes de encuestas en el área médica, en 117 estudios publicados, se encontró que muchos criterios se reportaron deficientemente: pocos estudios proporcionaron la encuesta o las preguntas centrales (35\%), informaron la validez o confiabilidad del instrumento (19\%), definieron la tasa de respuesta $(25 \%)$, discutieron la representatividad de la muestra (11\%) o identificaron cómo manejaron los datos faltantes (11\%); otros investigaciones encontraron resultados similares $4,7,8$. No se encontraron estudios que evalúen la calidad de los informes de encuestas en el área de Odontología.

Katrack et al. ${ }^{9}$, en una revisión sistemática, encontró más de 193 herramientas para evaluar calidad de los informes de investigación publicados. Actualmente, en la Red EQUATOR (www.equator-network.org) se pueden encontrar muchas de estas herramientas o guías para redactar buenos informes de investigaciones en salud; algunos de ellos, como la guía STROBE y STARD, permiten mejorar la calidad de los informes de estudios observacionales y de precisión diagnóstica respectivamente, y aunque muchas veces emplean cuestionarios, estas directrices no son específicas para orientar la descripción de los métodos y características de los informes de resultados que son exclusivos de encuestas ${ }^{10}$. El 2004 se publicó la lista de verificación CHERRIES que permite valorar las encuestas electrónicas y el 2014 aparece una nueva propuesta: las directrices SURGE para encuestas no web ${ }^{10-12}$. Adicionalmente a estas guías, han surgido otras propuestas, sin embargo, la gran mayoría de las herramientas de evaluación de calidad de los informes de estudios que utilizan encuestas no se han desarrollado con suficiente atención a los criterios de validez, por lo que no han sido respaldadas internacionalmente como completamente suficientes para evaluar la calidad de los informes de estos estudios ${ }^{5,13-16}$. En consecuencia, actualmente no existe un consenso en el uso de estas herramientas, lo que repercute en que muchas investigaciones basadas en encuestas no utilicen metodologías rigurosas o "mejores prácticas" en su diseño ${ }^{17-21}$.

Los estudios por encuestas demandan los mismos estándares de práctica de investigación al igual que cualquier otro. Por lo que esta nota científica tiene por objetivo presentar las directrices más aceptadas que ayuden a identificar los criterios de calidad clave para mejorar y evaluar la comunicación de este tipo de estudios y a la vez sean una guía práctica para investigadores que deseen realizar este tipo de trabajos con altos estándares y un valor real.

\section{El método de encuesta}

Son muchas las formas de emplear el método de encuesta, su selección dependerá del enfoque en el que esté orientado el trabajo de investigación y la pregunta que se desea responder, además de las condiciones propias del mismo investigador (como el tiempo y recursos económicos). Las encuestas son herramientas de investigación poderosas que transmiten información valiosa sobre tendencias de enfermedades, factores de riesgo, resultados de tratamiento, calidad de vida, satisfacción de la atención, evaluaciones educativas, además de explorar creencias, actitudes, comportamientos, mercadeo, políticas y opinión pública ${ }^{2,5}$. Los métodos más usados son la entrevista face to face, vía electrónica, telefónica, los cuestionarios administrados o autoministrados, estructurados (preguntas cerradas) o no estructurados (preguntas abiertas) ${ }^{5,7}$.

La selección del método es importante, porque de ello dependerá el éxito del proceso de recolección de datos; existe evidencia que sugiere que el método directo tiene mejor tasa de respuesta que aquel realizado vía web, de la misma forma el cuestionario autoadministrado ayuda a minimizar el sesgo de confianza ${ }^{17}$.

Diman et al. ${ }^{22}$ identifican cuatro errores en los métodos de encuesta: el error de cobertura, error de muestreo, error de medición y error de no respuesta, los cuales se pueden controlar utilizando estrategias para reducir el error, como: uso de diseńo multimodal cuando se desconoce la población total e individuos incluidos en la muestra; identificar claramente a la población de interés mediante estrategias de reclutamiento, aumentando el tamaño de muestra y realizando muestreo aleatorizado; utilizar instrumentos válidos y confiables, así como un diseño amigable con características visuales llamativas 
para los encuestados y procedimientos de seguimiento para aquellos que no responden.

Todos los estudios por encuesta deben seguir las normas de buenas prácticas para investigación científica, respetando la autonomía de los participantes. La investigación debe regirse de acuerdo al reglamento ético de su institución, código de ética profesional y normas nacionales e internacionales que puedan verse comprometidas. El investigador tiene la obligación de guardar la confidencialidad de los datos y respetar los procedimientos legales adheridos a estos ${ }^{23}$.

\section{Calidad en el estudio por encuesta}

La evaluación de la calidad de los estudios se hizo importante a partir del enfoque de la Medicina basada en la evidencia, que se centró en identificar, evaluar y sintetizar la investigación médica, la cual se extendió posteriormente a otras disciplinas como la Odontología y las ciencias del comportamiento y sociales ${ }^{2}$.

La calidad de un estudio depende de que los investigadores hayan tomado las medidas adecuadas para maximizar la validez y minimizar el sesgo en sus hallazgos. Además, está relacionada con el producto final (si es bueno como tal) y con el proceso de la encuesta; es decir, es importante perseguir ambos, la calidad del proceso y la calidad del producto ${ }^{15,24}$.

Los estudios de mayor calidad tienen menos probabilidad de incluir errores, o es más probable que informen de forma clara y transparente sobre los errores y limitaciones del estudio permitiendo así una mejor valoración de sus hallazgos y conclusiones ${ }^{7,8,25,26}$. La evaluación de la calidad del estudio tiene varias ventajas, como identificar las fortalezas y debilidades en la evidencia, proporcionando recomendaciones para intervenciones, políticas y prácticas, y para mejorar la investigación y estándares de publicación.

\section{Guías actuales para la evaluación de calidad de encuestas}

El alto número de estudios basados en encuestas en la literatura científica actual conlleva la necesidad de evaluar críticamente la utilidad y validez de los resultados de estas investigaciones. De allí la importancia del uso de las guías o directrices de reporte, las cuales son herramientas simples y estructuradas que guían la redacción de manuscritos. Una directriz proporciona una lista mínima de información necesaria para garantizar que un manuscrito pueda ser entendido por un lector, replicado por un investigador, incluido en una revisión sistemática y usado por un médico para tomar una decisión clínica ${ }^{10}$.

Los informes rigurosos, elaborados de acuerdo a estas guías, previenen interpretaciones erróneas y aplicaciones inadecuadas que podrían causar daño a los pacientes. Además, seguir estas directrices puede ayudar a los editores y revisores a mantener un proceso de revisión enfocado de alta calidad. Por lo tanto, hay una creciente necesidad que las revistas respalden estas directrices y refieran a los autores a utilizar listas de comprobación de informes especializados que sean pertinentes al diseño de su estudio.

A continuación, describimos las dos principales directrices para informes de investigaciones basadas en encuestas: SURGE y CHERRIES (Tabla 1).

Tabla 1. Características principales de las directrices SURGE y CHERRIES

\begin{tabular}{|c|c|}
\hline Asunto & SURGE (2014) \\
\hline Propósito & $\begin{array}{l}\text { Está dirigido primariamente para investigaciones que emplean encuestas } \\
\text { postales autoadministradas. }\end{array}$ \\
\hline Aplicación & $\begin{array}{l}\text { Diseñado para informar resultados de la investigación de encuestas de salud } \\
\text { y servicios de salud. Por extensión, las directrices pueden ser adecuadas para } \\
\text { informar investigaciones de encuestas en otras áreas, como sicología, sociología, } \\
\text { etc. }\end{array}$ \\
\hline Limitaciones & Excluye las encuestas en persona, vía telefónica, basadas en la web y otras. \\
\hline $\begin{array}{l}\text { Proceso de } \\
\text { elaboración }\end{array}$ & $\begin{array}{l}\text { Para su validación se siguieron los siguientes pasos: } \\
\text { - Búsqueda bibliográfica sobre directrices existentes, criterios y/o investigación } \\
\text { empírica sobre la calidad de los informes de encuestas. Incluyó: una revisión } \\
\text { sistemática de la literatura científica publicada y una búsqueda de instrucciones } \\
\text { para autores en revistas médicas. } \\
\text { - Desarrollo y prueba piloto de un conjunto de criterios de evaluación de cali- } \\
\text { dad para informes de investigaciones de encuestas } \\
\text { - Aplicación de los criterios de evaluación de calidad a una muestra representa- } \\
\text { tiva de artículos de investigación de encuestas }\end{array}$ \\
\hline
\end{tabular}

Estado actual Se ha puesto a disposición de la comunidad científica para su aplicación y evaluación. El grupo que elaboró las directrices seguirá evaluando su utilidad e impacto. Aparece en la guía para informes de investigación en salud, junto con otras listas de comprobación propuestas por la Red EQUATOR y Wiley Blackwell.

Dirigido primariamente a encuestas basadas
en la web (cuestionarios electrónicos administrados en Internet o una Intranet).

También es válido para encuestas administradas vía correo electrónico.

No utiliza una tasa única de respuesta sino tres métricas de respuestas.

No se describe.

Esta directriz aparece en la Red EQUATOR; además, algunas revistas, como Journal of Medical Internet Research y Journal of Pediatrics, solicitan $a$ los autores que envíen manuscritos a su revista considerando estas directrices. 


\section{El instrumento SURGE}

Las directrices para informes de encuestas, denominado SURGE (SUrvey Reporting GuidelinE), fueron elaboradas para orientar y mejorar la calidad de la presentación de informes de investigación donde la información recogida ha sido por medio de encuestas postales autoadministradas ${ }^{12}$. Estas directrices, a comparación de otras también dirigidas a investigaciones de encuestas, se encuentran en un proceso muy avanzado de validación. Han seguido la guía para el desarrollo de directrices de informes propuestos por Moher et al. ${ }^{26}$ y consideramos que ya pueden utilizarse para el fin por el cual fueron creadas puesto que no se cuenta con otro instrumento validado. Es posible que en los próximos años aparezca una versión actualizada de estas directrices, con algunas pequeñas modificaciones a la versión actual, producto de una evaluación de su aplicación por la comunidad científica; sin embargo, tal como está, SURGE es útil para los lectores, autores, revisores y editores que elaboran o evalúan informes de encuestas. Existen algunos estudios ${ }^{4,8}$ que han evaluado la calidad de los informes de encuestas en la literatura médica, aplicando como criterio de evaluación SURGE, concluyendo que existe una calidad deficiente de estos informes. Algunas de sus características se presentan en la Tabla 1.

Las directrices SURGE incluyen una lista de 33 ítems, los cuales evalúan seis aspectos de los informes de investigación de encuestas: el título y resumen, la introducción, los métodos, los resultados, la discusión y los indicadores de calidad ética (Tabla 2).

Tabla 2. Lista de comprobación SURGE

\begin{tabular}{lll}
\hline Sección & Ítem \\
\hline
\end{tabular}

Título y resumen

1 ¿Se declara el diseño del estudio en el título y/o resumen?

Introducción

2 ¿Hay una explicación de por qué es necesaria la investigación, colocando el estudio en el contexto de trabajos previos en campos relevantes?

3 ¿Se explica el propósito u objetivo del artículo?

Métodos

Instrumento de investigación

Selección de la muestra

Administración de la encuesta

Análisis

\section{Resultados}

$4 \quad$ ¿Se describe el cuestionario?

5 Si se utilizó un instrumento existente, ¿se presentaron sus propiedades sicométricas?

6 Si se utilizó un instrumento existente, ¿se proporcionan las referencias al trabajo original?

7 Si se utilizó un instrumento nuevo, ¿se mencionan los procedimientos utilizados para desarrollarlo y realizar una prueba piloto?

8 Si se utilizó un instrumento nuevo, ¿se informó su validez y confiabilidad?

9 ¿Se proporciona una descripción de los procedimientos de puntuación?

10 ¿Hay una descripción de la población de estudio y el marco muestral utilizado para identificar esta población?

11 ¿Los autores proporcionan una descripción de cuán representativa es la muestra respecto a la población?

12 ¿Se presenta un cálculo del tamaño de la muestra o una justificación del tamaño de la muestra?

13 ¿Cuál fue el modo de administración?

¿Los autores proporcionan información sobre el tipo de contacto y cuántos intentos fueron hechos para contactar datorio)

15 ¿Los autores informan si se proporcionaron incentivos (financieros $\mathrm{u}$ otros)?

16 ¿Hay una descripción de quién se acercó a los participantes potenciales? (por ejemplo, identificación de quién firmó la carta de presentación)

17 ¿Se describe el método de análisis de los datos?

18 ¿Los autores proporcionan los métodos para el análisis del error de no respuesta?

19 ¿Se proporciona el método para calcular la tasa de respuesta?

20 ¿Se proporcionan definiciones para terminaciones completas versus parciales?

21 ¿Se proporcionan los métodos para manejar los datos perdidos de los ítems? 
Tabla 2. Continuación

\begin{tabular}{lcl}
\hline Sección & Ítem & \multicolumn{1}{c}{ Recomendación } \\
\hline 23 & ¿Se tienen en cuenta todos los encuestados? \\
24 & ¿Se proporciona información sobre cómo los no encuestados difieren de los encuestados? \\
25 & ¿Los resultados se presentan claramente? \\
26 & ¿Los resultados reflejan los objetivos del estudio?
\end{tabular}

Discusión

\begin{tabular}{lll} 
& 27 & ¿Se resumen los resultados en relación a los objetivos del estudio? \\
& 28 & ¿Se mencionan las fortalezas del estudio? \\
& 29 & ¿Se indican las limitaciones del estudio (teniendo en cuenta las potenciales fuentes de sesgo o imprecisión)? \\
& 30 & ¿Existe una discusión explícita de la generalización (validez externa) de los resultados? \\
$\begin{array}{l}\text { Indicadores de } \\
\text { calidad ética }\end{array}$ & & \\
& 31 & ¿Se informa sobre el financiamiento del estudio? \\
& 32 & ¿Se informa sobre la revisión del estudio por un Comité de Ética en Investigación? \\
& 33 & ¿Se informa sobre los procedimientos del consentimiento de los individuos? \\
\hline
\end{tabular}

Fuente: traducido de Grimshaw ${ }^{12}$

Para una comprensión más clara de las directrices SURGE se explicará algunas de ellas, específicamente las relacionadas a la sección de Métodos. Sobre el instrumento de medición (cuestionario), los ítems 5 y 6 hacen mención a que si en el estudio se aplica un instrumento ya existente, entonces se debe informar sus propiedades sicométricas: confiabilidad (consistencia en los resultados de una medición a otra), validez (precisión en la medición del concepto), y en el caso de estudios longitudinales, sensibilidad al cambio ${ }^{5}$. Además, debería mencionarse la cita bibliográfica que evidencia la validación de dicho instrumento.

Los ítems 7 y 8 indican que si en el estudio se aplica un instrumento nuevo entonces se debe mencionar explícita y claramente los procedimientos que se siguieron para elaborar el instrumento y probarlo en una muestra piloto.

Respecto a la selección de la muestra, las directrices recomiendan identificar la población de estudio señalando el marco muestral empleado (lista de todos los miembros de la población a ser encuestada y de la cual se extrae la muestra) (ítem 10). Además, describir la representatividad de la muestra (ítem 11), la cual está relacionada con el método de selección de los participantes, y mencionar cómo se determinó el tamaño de la muestra (ítem 12): a criterio del investigador (justificando el motivo) o por medio de una fórmula (especificando los valores considerados en el mismo).

Sobre la administración de la encuesta, SURGE recomienda indicar el modo de administración (ítem 13): en persona, vía mail o mixta.

\section{El instrumento CHERRIES}

La lista de comprobación para informar resultados de encuestas electrónicas por Internet, denominado CHERRIES (Checklist for reporting results of Internet e-surveys) es una lista validada que busca asegurar una descripción completa de la metodología de las encuestas basadas en la web (cuestionarios en línea) (Tablas 1 y 3 ). Fue propuesta en el 2004 dentro de un editorial del Journal of Medical Internet Research ${ }^{11}$, posteriormente se realizó una pequeña corrección el $2012^{27}$.

Existe mucho debate sobre la validez de las investigaciones que emplean encuestas Web ya que pueden estar sujetas a muchos sesgos debido a que emplean muestras por conveniencia; además presentan varias desventajas: una tasa de respuesta muy baja, sus conclusiones son limitadas y muchas revistas suelen rechazar con facilidad estos estudios; sin embargo, son útiles para: generar hipótesis, emplearlas como pruebas piloto de un cuestionario y realizar un experimento basado en la Web ${ }^{11}$. Por ello, es importante que los investigadores presenten los informes provenientes de estos estudios teniendo en cuenta la guía CHERRIES (Tabla 3).

\section{Conclusiones}

La investigación de encuestas es útil y legítima y su uso dependerá del enfoque de la pregunta de investigación, sus principales beneficios radican en que ayudan a describir, explorar y realizar construcciones de variables de interés y pese a que tienen potencial para una variedad de fuentes de error, existen varias estrategias para reducir estos sesgos. La evaluación de la calidad de los estudios por encuestas debe ser una práctica importante para promover una mayor precisión, transparencia y evaluación de la investigación en Odontología. Actualmente se cuenta con las guías SURGE y CHERRIES que brindan pautas para realizar informes de calidad, especialmente sobre los aspectos metodológicos básicos para este tipo de estudios. Se requieren estudios que evalúen la calidad de los informes de investigaciones basadas en encuestas en el área de Odontología. 
Tabla 3. Lista de comprobación CHERRIES

\begin{tabular}{l} 
Categoría / Ítem \\
\hline Diseño \\
Describa el diseño del estudio \\
Comité de Ética en Investigación \\
(CEI) aprobó e informó el proceso \\
de consentimiento
\end{tabular}

Aprobación del CEI

Consentimiento informado

Protección de datos

Desarrollo y prueba piloto

Desarrollo y pruebas

\section{Proceso de reclutamiento y descrip- ción de la muestra que tiene acceso al cuestionario}

Encuesta abierta frente a encuesta cerrada

Modo de contacto

Publicidad de la encuesta

\section{Administración de la encuesta}

Web/correo electrónico

Contexto

Obligatorio / voluntario

Incentivos

Tiempo / fecha

Aleatorización de ítems o cuestionarios

Preguntas adaptativas

Número de ítems

Número de pantallas (páginas)

Verificación de integridad

Paso de revisión
Explicación

Describa la población objetivo y marco muestral. ¿Es una muestra por conveniencia? (esto es más probable en encuestas "abiertas")

Mencione si el estudio fue aprobado por un CEI.

Describa el proceso de consentimiento informado. ¿Dónde se les dijo a los participantes la duración de la encuesta, qué datos se almacenaron y dónde y por cuánto tiempo, quién fue el investigador y el propósito del estudio?

Si se recopiló o almacenó información personal describa qué mecanismos fueron utilizados para proteger el acceso no autorizado.

Indique cómo se desarrolló la encuesta, incluso si la usabilidad y funcionalidad técnica del cuestionario electrónico se había probado antes de responder el cuestionario.

Una "encuesta abierta" es una encuesta disponible para cada visitante de un sitio web, mientras que una encuesta cerrada solo está disponible a una muestra que el investigador conoce (encuesta protegida por contraseña).

Indique si el contacto inicial con los participantes potenciales fue hecho o no en Internet. (Los investigadores también pueden enviar cuestionarios por correos y permitir la entrada de datos basada en la web).

¿Cómo/dónde se anunció o publicitó la encuesta? Algunos ejemplos son medios de comunicación fuera de línea (periódicos) o en línea (listas de correo - en caso afirmativo ¿cuáles?) o pancarta publicitando el anuncio de la encuesta (¿dónde se publicaron estos anuncios y cómo se veían?). Es importante conocer la redacción del anuncio, ya que influirá mucho quién elige participar. Idealmente el anuncio de la encuesta debería publicarse como un apéndice.

Indique el tipo de encuesta electrónica (por ejemplo, una publicada en un sitio web o una enviada a través de correo electrónico). Si se trata de una encuesta por correo electrónico ¿se ingresaron las respuestas manualmente en una base de datos o hubo un método automático para capturar las respuestas?

Describa el sitio web (para la lista de correo / grupo de noticias) en el que se publicó la encuesta. ¿De qué se trata el sitio web, quién lo visita, qué es lo que están buscando normalmente los visitantes? Discuta hasta qué punto el contenido del sitio web podría preseleccionar la muestra o influir en los resultados. Por ejemplo, una encuesta sobre vacunación en un sitio web, antiinmunización tendrá resultados diferentes de una encuesta web realizada en un sitio web del gobierno.

¿Era una encuesta obligatoria para ser completada por cada visitante que quería ingresar al sitio web o fue una encuesta voluntaria?

¿Se ofrecieron incentivos? (por ejemplo, incentivos monetarios, premios, o no monetarios, tal como, un ofrecimiento para proporcionar los resultados de la encuesta)

¿En qué plazo se recogieron los datos?

Indique si los ítems se aleatorizaron o alternaron para evitar los sesgos.

Indique si se utilizaron preguntas adaptativas (ciertos ítems solo se muestran condicionalmente en función de las respuestas a otros ítems) para reducir el número y complejidad de las preguntas.

¿Cuál fue el número de ítems del cuestionario por página? El número de ítems es un factor importante para la tasa de finalización.

¿En cuántas páginas se distribuyó el cuestionario? El número de páginas es un factor importante para la tasa de finalización.

¿Es técnicamente posible hacer verificaciones de consistencia o integridad (llenado de todos los campos) antes que se envíe el cuestionario? ¿Se hizo esto?, y en caso afirmativo, cómo (usualmente JavaScript). Una alternativa es verificar después que el cuestionario se ha enviado (y resaltar los ítems obligatorios). Si esto se ha hecho, debe ser reportado. Todos los ítems deben proporcionar una opción de no respuesta como "no aplicable" o "prefiero no especificarlo", y la selección de una opción de respuesta debe hacerse cumplir.

Indique si los encuestados pudieron revisar y cambiar sus respuestas (por ejemplo, a través de un botón Atrás o un paso de Revisión que muestra un resumen de las respuestas y pregunta a los encuestados si están en lo correcto). 
Tabla 3. Continuación

\begin{tabular}{ll}
\hline Categoría / Ítem & Explicación \\
\hline Tasas de respuesta &
\end{tabular}

Tasas de respuesta

Visitante único del sitio

Tasa de visualización (razón de visitantes de encuestas únicas / visitantes únicos del sitio)

Tasa de participación (Razón de visitantes únicos que aceptaron participar / visitantes únicos de la primera página de la encuesta).

Tasa de finalización (razón de usuarios que terminaron la encuesta / usuarios que aceptaron participar)

\section{Prevención de entradas múltiples} del mismo individuo

Cookies utilizados

Control del IP*

Análisis de archivo. $\log$ (de registro)

Registro

\section{Análisis}

Manejo de cuestionarios incompletos

¿Solo se analizaron los cuestionarios completados? ¿Fueron también analizados los cuestionarios que terminaron temprano? (Donde, por ejemplo, los usuarios no respondieron todas las páginas del cuestionario).

Cuestionarios enviados con una marca de tiempo atípica

Algunos investigadores pueden medir el tiempo que las personas necesitan para completar un cuestionario y excluir cuestionarios que se enviaron demasiado pronto. Especifique el plazo que se usó como punto de corte y describa cómo se determinó este punto.

Corrección estadística
Si proporciona tasas de visualización o tasas de participación, debe definir cómo determinó un visitante único. Existen diferentes técnicas disponibles, basadas en direcciones IP o cookies o ambos.

Requiere contar visitantes únicos a la primera página de la encuesta, divididos por el número de visitantes únicos del sitio (¡no visitas a la página!). No es inusual tener tasas de visualización de menos del $0,1 \%$ si la encuesta es voluntaria.

Cuente el número único de personas que completaron la primera página de la encuesta (o aceptaron participar, por ejemplo, marcando una casilla de verificación), dividida por el número de personas que visitaron la primera página de la encuesta (o la página de consentimiento informado, si está presente). Esta también se puede llamar "tasa de reclutamiento".

El número de personas que enviaron la última página del cuestionario, dividido por el número de personas que aceptaron participar (o enviaron la primera página de la encuesta). Esto solo es relevante si hay una página separada de "consentimiento informado" o si la encuesta abarca varias páginas. Esta es una medida del nivel de abandono de la encuesta. Tenga en cuenta que "finalización" puede implicar dejar ítems del cuestionario en blanco. Esta no es una medida de cuán completamente se llenaron los cuestionarios. Si necesita una medida para esto, use la palabra "tasa de integridad".

Indique si se utilizaron cookies para asignar un identificador de usuario único a cada computadora cliente. Si es así, mencione la página en la que se configuró y leyó la cookie, y durante cuánto tiempo fue válida la cookie. ¿Se evitaron las entradas duplicadas evitando que los usuarios accedan a la encuesta dos veces? o ¿se eliminaron las entradas duplicadas de la base de datos con la misma ID de usuario antes del análisis? En este último caso, qué entradas fueron guardadas para el análisis? (por ejemplo, la primera entrada o la más reciente).

Indique si se utilizó la dirección IP de la computadora cliente para identificar posibles entradas duplicadas del mismo usuario. Si es así, mencione el periodo de tiempo por el que no se permitieron dos entradas de la misma dirección IP (por ejemplo, 24 horas). ¿Se evitaron las entradas duplicadas al evitar que los usuarios con la misma dirección IP accedan a la encuesta dos veces; o eran entradas duplicadas de la base de datos que tenían la misma dirección IP en un periodo determinado antes del análisis? Si fue lo último, ¿qué entradas se guardaron para el análisis? (por ejemplo, la primera entrada o la más reciente).

Indique si se utilizaron otras técnicas para analizar el archivo de registro para la identificación de entradas múltiples. Si es así, descríbalo.

En las encuestas "cerradas" los usuarios deben iniciar sesión primero y es más fácil evitar entradas duplicadas del mismo usuario. Describa cómo se hizo esto. Por ejemplo, ¿la encuesta nunca se mostró por segunda vez una vez que el usuario la completó, o el nombre de usuario se almacenó junto con los resultados de la encuesta y luego se eliminó? Si es lo último, ¿qué entradas se guardaron para el análisis?, por ejemplo, ¿la primera entrada o la más reciente?

\section{*IP: Internet Protocol \\ Fuente: traducido de Eysenbach ${ }^{11}$}

\section{Referencias bibliográficas}

1. LaVange LM, Koch GG, Schwartz TA. Applying simple survey methods to clinical trials data. Statist Med. 2001;20:2609-2623. DOI: 10.1002/sim.732.

2. Ponto J. Understanding and evaluating survey research. J Adv Pract Oncol 2015;6(2):168-171.

3. Bennett C, Khangura S, Brehaut JC, Graham ID, Moher $\mathrm{D}$, et al. Reporting guidelines for survey research: an analysis of published guidance and reporting practices. PLoS Med. 2011;8(8):e1001069. DOI: 10.1 371/journal.pmed.1001069.
4. Turk T, Elhady MT, Rashed S, Abdelkhalek M, Nasef SA, Khallaf et al. Quality of reporting web-based and non-webbased survey studies: What authors, reviewers and consumers should consider. PLoS One. 2018 Jun 18;13(6):e0194239. DOI: 10.1371/journal.pone.0194239.

5. Kelley K, Clark B, Brown V, Sitzia J. Good practice in the conduct and reporting of survey research. Int J Qual Health Care. 2003;15(3):261-6. DOI: 10.1093/intqhc/mzg031.

6. Cornesse C, Bosnjak M. Is there an association between survey characteristics and representativeness? A meta-analysis. Surv Res Methods. 2018;12(1):1-13. DOI:10.18148/srm/2018.v12i1.7205. 
7. Li AH, Thomas SM, Farag A, Duffett M, Garg AX Naylor KL. Quality of survey reporting in nephrology journals: a methodologic review. Clin J Am Soc Nephrol. 2014 Dec 5; 9(12):2089-94. DOI: 10.2215/ CJN.02130214.

8. Pagano MB, Dunbar NM, Tinmouth A, Apelseth TO, Lozano M, Cohn CS, et al. A methodological review of the quality of reporting of surveys in transfusion medicine. Transfusion. 2018;58(11):2720-27. DOI: 10.1111/trf.14937.

9. Katrak P, Bialocerkowski A, Massy-Westropp N, Saravana Kumar VS, Grimmer K. A systematic review of the content of critical appraisal tools. BMC Med Res Methodol. 2004;4:22. DOI:10.1186/1471-2288-4-22.

10. EQUATOR Network. Library for Health Research Reporting. [Consultado el 3 de setiembre 2019]. Accesible en: http://www.equator-network.org/.

11. Eysenbach G. Improving the quality of web surveys: the checklist to report the results of electronic surveys online (CHERRIES). J Med Internet Res. 2004;6(3):e34.

12. Grimshaw J. SURGE (The SUrvey Reporting GuidelinE). Guidelines for reporting health research: a user's manual. 2014:206-13.

13. Draugalis JR, Coons SJ, Plaza CM. Best practices for survey research reports: a synopsis for authors and reviewers. Am J Pharm Educ. 2008;72(1):11. DOI: 10.5688/ aj720111.

14. Huston P. Reporting on surveys: information for authors and peer reviewers. Can Med Assoc J. 1996;154(11):1695-1704.

15. Artino AR, La Rochelle J, Dezee K, Gehlbach H. Developing questionnaires for educational research. Med Teach. 2014,36(87):463-474.

16. Qin Liu. A survey data quality strategy: the institutional research perspective. IR Application. 2012;34:1-9.

17. Artino AR, Phillips AW, Utrankar A, Durning SJ. "The questions shape the answers": assessing the quality of published survey instruments in health professions education research. Acad Med. 2018;93(3):456-463. DOI: 10.1097/ACM.0000000000002002.
18. Parrish DO. RE: Good decisions cannot be made from bad surveys. Mil Med. 2017;182(5):1590. DOI: 10.7205 / MILMED-D-17-00044.RE.

19. Souza AC, Alexandre NMC, Guirardello EB. Psychometric properties in reliability and validity assessment instruments. Epidemiol Serv Saude. 2017 Jul-Sep; 26(3):649659. DOI: 10.5123 / S1679-49742017000300022.

20. Funkhouser E, Vellala K, Baltuck C, Cacciato R, Durand E, McEdward D, et al. National Dental PBRN Collaborative Group. Survey methods to optimize the response rate in the national research network based on dental practice. Eval Health Prof. 2017 Sep; 40(3):332-358. DOI: $10.1177 / 0163278715625738$.

21. Langbecker D, Caffery LJ, Gillespie N. Smith AC. Using survey methods in telehealth research: a practical guide. J Telemed Telecare. 2017 Oct; 23(9):770-779. DOI: 10.1177 / $1357633 X 17721814$.

22. Dillman DA., Smyth JD, Christian LM. Internet, phone, mail, and mixed-mode surveys: the tailored design method. 4th ed. Hoboken, NJ: John Wiley \& Sons, Inc. 2014 .

23. World Health Organization (WHO). Handbook for good clinical research practice (GCP): Guidance for implementation. Geneva: WHO; 2005.

24. World Health Organization. Oral Health Surveys, 4rd ed. Geneva, Switzerland: WHO; 1997.

25. Duffett M, Burns KE, Adhikari NK, Arnold DM, Lauzier F, Kho ME, et al. Quality of survey reports in critical care journals: a methodological review. Crit Care Med. 2012;40(2):441-9. DOI: 10.1097/CCM.0b013e$318232 \mathrm{~d} 6 \mathrm{c} 6$.

26. Moher D, Schulz K, Simer I, Altman D. Guidance for developers of health research reporting guidelines. PLoS Med. 2010; 7(2):e1000217. DOI: 10.1371/journal. pmed.1000217.

27. Eysenbach G. Correction: improving the quality of Web surveys: the checklist for reporting results of Internet e-surveys (CHERRIES). J Med Internet Res. 2012;14(1):e8. DOI: 10.2196/jmir.2042. 